\title{
Theoretical study of the electronic spectrum of magnesium-porphyrin
}

\author{
Mercedes Rubio and Björn O. Roos ${ }^{\text {a) }}$ \\ Department of Theoretical Chemistry, Chemical Center, University of Lund, P.O.B. 124, \\ S-221 00 Lund, Sweden \\ Luis Serrano-Andrés and Manuela Merchán \\ Departamento de Química Física, Universitat de València, Av. Dr. Moliner 50, Burjassot, \\ E-46100 Valencia, Spain
}

(Received 29 September 1998; accepted 12 January 1999)

\begin{abstract}
Multiconfigurational self-consistent field (SCF) and second order perturbation methods have been used to study the electronic spectrum of magnesium-porphyrin $(\mathrm{MgP})$. An extended ANO-type basis set including polarization functions on all heavy atoms has been used. Four allowed singlet states of $E_{1 u}$ symmetry have been computed and in addition a number of forbidden transitions and a few triplet states. The results lead to a consistent interpretation of the electronic spectrum, where the $Q$ band contains one transition, the $B$ band two, and the $N$ band one. The computed transition energies are consistently between 0.1 and 0.5 too low compared to the measured band maxima. The source of the discrepancy is the approximate treatment of dynamic correlation (second order perturbation theory), limitations in the basis set and the fact that all measurements have been made on substituted magnesium porphyrins. (C) 1999 American Institute of Physics. [S0021-9606(99)30814-X]
\end{abstract}

\section{INTRODUCTION}

The spectroscopic properties of porphyrins have attracted attention experimentally and theoretically since the middle of the century. The great biological importance of these compounds motivated the initial interest, which has been subsequently enlarged by their potential technological applications. ${ }^{1,2}$ Thus, for instance, the linear and nonlinear optical properties of systems based on porphyrins are at present studied as possible materials for the design of optical devices. $^{3-5}$

As regards the role porphyrins play in biological systems, one could mention their participation in processes such as oxygen and electron transport. Moreover, chlorophyll (in green plants) and bacteriochlorophyll (in photosynthetic bacteria) molecules take part in the primary events of photosynthesis, absorbing light, and carrying the electronic excitation energy to the so called "reaction center," where charge separation occurs involving a few special porphyrin molecules. ${ }^{6}$ The importance of photosynthesis has lead to numerous studies involving porphyrins, not only to understand the initial steps of this process, essential for all living organisms, but also to try to develop artificial systems which are able to mimic it (see, for example, Refs. 7-9, and therein).

The first systematic studies on the absorption and emission spectra of metal-porphyrins were performed in order to attempt to correlate the changes observed with the nature of the bonding between the metal and the macrocycle. ${ }^{10,11}$ The effects of the substituents on the macrocycle in the visible and near-UV (ultraviolet) absorption bands were rationalized by Gouterman at the end of the fifties. ${ }^{12} \mathrm{He}$ also explained the differences between the spectra of free base porphins and

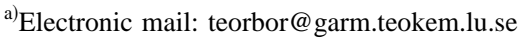

metal-porphyrins using the cyclic polyene model introduced by Simpson. ${ }^{13}$ The electronic absorption spectra of metalporphyrins have common features. Thus, the absorption is very weak in the visible region where the so called $Q$ band appears showing vibrational structure. The most intense absorption occurs in the near-UV region and it is known as the $B$ or Soret band. The energy, intensity, and vibrational pattern of the $Q$ band are most strongly affected by the central metal. ${ }^{10}$ In free base porphins, in which the central metal is replaced by two hydrogen atoms, the most striking change is the splitting of the $Q$ bands due to the loss of square symmetry. Very little splitting is, however, observed in the $B$ band. The different intensities of the $Q$ and $B$ bands was first explained by Gouterman's model for the excited states of porphyrins. ${ }^{12}$ This model is based on a four-configurational description of the states, involving the two highest occupied and the two lowest virtual orbitals. Weiss et al. ${ }^{14}$ performed in 1965 Pariser-Parr-Pople (PPP) calculations on the singlet and triplet states of porphyrins, which gave support to the four-orbital model. At the same time, the absorption spectra of substituted metal-porphyrins were measured in solution by Caughey et al., ${ }^{15}$ extending the energy range previously studied. ${ }^{10}$ Two weak bands were detected in the UV region down to $250 \mathrm{~nm}$ for which Caughey et al. ${ }^{15}$ introduced the generic names $N$ and $L$. The four main bands $(Q, B, N$, and $L$ ) were then assigned on the basis of the PPP calculations of Weiss et al. ${ }^{14}$ As far as we know, the first and most extensive investigation on the spectroscopic properties of porphyrins in gas phase was performed by Edwards et al. ${ }^{16,17}$ They studied the gas-phase absorption spectra of octalkylporphyrins ${ }^{16}$ and tetraphenylporphyrins ${ }^{17}$ in the energy range 800-200 nm (1.5-6.2 eV), identifying a new high energy band, $M$ band, around $6 \mathrm{eV}$. On the other hand, transient absorption spectroscopy has also been applied to metal-porphyrins in order to determine the spectral features which characterize the dif- 
ferent types of excited states $\left(\pi \rightarrow \pi^{*}\right.$, charge transfer metalring, and metal $(d, d)$ states). ${ }^{18}$

Many theoretical studies have been devoted to the excited states of porphyrins. A short review can be found in Ref. 19, which also reports a semiempirical CS INDO CI (configuration interaction) study of the electronic spectra of free base porphin, chlorin, and their magnesium derivates. As examples of how the interpretations of the electronic spectrum depend on the parameters used in the semiempirical approach, we could cite the study of McHugh et al. ${ }^{20}$ These authors extended the previous PPP investigation carried out by Weiss et al. ${ }^{14} \mathrm{~A}$ conclusive assignment for the $B$ and higher energy bands of the electronic spectrum of free base porphin could not be provided from their results. ${ }^{20}$ The interpretation of the near-UV and UV region has actually been matter of controversy until today. Thus, in general, theoretical studies agree in assigning the $Q$ bands to two $\pi$ $\rightarrow \pi^{*}$ electronic transitions qualitatively described within the four-orbital model, ${ }^{12}$ which become degenerate in metalporphyrins due to the higher symmetry. The number of $\pi$ $\rightarrow \pi^{*}$ states which are responsible for the $B$ and $N$ bands, however, has been debated. In particular, high level ab initio studies on the excited states of free base porphin (FBP), which only recently have been possible to perform, lead to different interpretations of the electronic spectrum. ${ }^{21-23}$

We have recently presented results from an investigation of the low-lying optically allowed valence excited states of FBP using multiconfigurational second-order perturbation (CASPT2) theory and a split-valence plus polarization basis set. ${ }^{23}$ According to our results, each band of the spectrum up to $4.5 \mathrm{eV}$ ( $Q, B, N$, and $L$ bands) is related to a pair of $\pi$ $\rightarrow \pi^{*}$ states. The interpretation is consistent with the experimental spectrum but differs from the assignments proposed on the basis of other types of ab initio calculations. ${ }^{21,22}$ For a detailed discussion see Ref. 23, which also includes a summary of the other $a b$ initio studies performed on the excited states of FBP. Most of the ab initio studies have been focused on FBP since the molecule can be considered as the basic unit from which porphyrins and their analogs derive.

In order to obtain more insight about the spectroscopic properties of porphyrins, we have now also carried out a study of the excited states of magnesium-porphyrin. This molecule is the metal-porphyrin related to chlorophylls. Apart from the substitution on the macrocycle, chlorophylls have one of the pyrrole rings reduced by two hydrogen atoms. In spite of these differences, common features still remain in the electronic spectra. ${ }^{24,25}$ Therefore, the characterization of the electronic spectrum of magnesium-porphyrin $(\mathrm{MgP})$ can contribute to a better understanding of spectra of chlorophylls. A detailed and conclusive assignment of the spectrum has not yet been achieved. As far as we know, two previous ab initio studies on the excited states of $\mathrm{MgP}$ have been reported. ${ }^{26,27}$ Small basis sets have been used in these studies, as well as a quite limited treatment of the electron correlation in one of them. ${ }^{26}$ In the present contribution, the CASPT2 method in combination with a basis set of splitvalence plus polarization quality has been used for the interpretation of the optical spectrum. In addition to the low-lying optically allowed valence excited states, the lowest forbidden
TABLE I. Symmetries of the $\pi \rightarrow \pi^{*}$ states in the $D_{4 h}$ and $D_{2 h}$ symmetry groups. $^{\mathrm{a}}$

\begin{tabular}{ccc}
\hline \hline Point group & $D_{4 h}$ & $D_{2 h}$ \\
\hline & $A_{1 g}, B_{1 g}$ & $A_{g}$ \\
$E_{u}$ & $B_{3 u}+B_{2 u}$ \\
& $A_{2 g}, B_{2 g}$ & $B_{1 g}$ \\
\hline \hline
\end{tabular}

$\mathrm{a}_{z}$ axis is perpendicular to the molecular plane.

states have been considered, including the lowest triplet states. An assignment for the $Q, B$, and $N$ bands is suggested, which is consistent with the experimental data and with the interpretation recently proposed for the electronic absorption spectrum of free base porphin. ${ }^{23}$

\section{COMPUTATIONAL DETAILS}

Generally contracted basis sets of atomic natural orbital (ANO) type have been used, which have been built from $\mathrm{Mg}(13 s 8 p 3 d) / \mathrm{C}, \mathrm{N}(10 s 6 p 3 d) / \mathrm{H}(7 s)$ primitive sets. ${ }^{28}$ The same contraction scheme $\mathrm{C}, \mathrm{N}[3 s 2 p 1 d] / \mathrm{H}[2 s]$ as in the study of free base porphin ${ }^{23}$ has been used in most calculations, together with the contraction $[4 s 3 p 1 d]$ for the magnesium atom.

An optimization of the geometry of the ground state of $\mathrm{MgP}$ was performed using density functional theory (DFT) at the B3PW91/6-31G* level, assuming $D_{4 h}$ symmetry. The equilibrium geometry predicted is in line with the results from other studies based also on density functional theory where a lower symmetry was used in the optimization process. ${ }^{29,30}$ The B3PW91/6-31G* optimized geometry has been used in all calculations.

The low-lying states of MgP have been computed using multiconfigurational second order perturbation theory through the CASPT2 method, ${ }^{31,32}$ which has shown to be especially powerful for studies of electronic spectra. ${ }^{33,34}$ The reference wave function and the molecular orbitals are determined using the complete active space self-consistent field (CASSCF) method. ${ }^{35}$ In the present study, the average CASSCF procedure has been used for all the states, except for the ground state. For technical reasons all calculations have been carried out in $D_{2 h}$ symmetry. The $\pi$-orbitals belong to the $b_{1 u}\left(a_{2 u}, b_{2 u}\right), b_{2 g}\left(e_{g}\right), b_{3 g}\left(e_{g}\right)$, and $a_{u}\left(a_{1 u}, b_{1 u}\right)$ irreducible representations of the $D_{2 h}\left(D_{4 h}\right)$ symmetry group, with the $z$ axis perpendicular to the molecular plane. Symmetry restrictions were imposed in the CASSCF calculations in order to prevent mixing between molecular orbitals belonging to different $D_{4 h}$ representations. Table I shows the relation between the irreducible representations of the $D_{4 h}$ and $D_{2 h}$ symmetry groups that the $\pi \rightarrow \pi^{*}$ states belong to. The investigation is mainly focused on the doubly degenerate $E_{u}$ states, which give rise to the optically allowed transitions. In most calculations only the $B_{3 u}$ component (see Table I) has been computed, since the same result was obtained in those cases where both components were considered. Therefore, the $D_{4 h}$ symmetry is expected to be preserved in all calculations, as test calculations have indicated. The $1 s$ core orbitals were kept frozen in the 
TABLE II. $\pi$-active spaces used in the calculations of the ground and excited states of Mg-Porphyrin.

\begin{tabular}{lll}
\hline \hline$(\text { klmn })^{\mathrm{a}}$ & $O / E^{\mathrm{b}}$ & $(\mathrm{CSF} / \text { determinants })^{\mathrm{c}}$ \\
\hline$(1111)$ & $4 / 4$ & $8 / 12$ \\
$(4332)$ & $12 / 16$ & $17865 / 61235$ \\
$(4333)$ & $13 / 18$ & $36022 / 127975$ \\
$(4442)$ & $14 / 16$ & $537705 / 2254521$ \\
$(4443)$ & $15 / 18$ & $1430940 / 6263425$ \\
\hline \hline
\end{tabular}

a $($ klmn $)$ stands for the number of $\pi$ orbitals in four of the irreducible representations of the $D_{2 h}$ point group $\left(b_{1 u}, b_{2 g}, b_{3 g}, a_{u}\right)$.

${ }^{\mathrm{b}}$ Number of orbitals/number of electrons.

${ }^{\mathrm{c} N u m b e r}$ of configuration state functions (CSF) and determinants for the CASSCF wave function of the ground state.

form determined by the ground state Hartree-Fock (HF) wave function and the $\sigma$ valence electrons were inactive, as well as the magnesium $2 s$ and $2 p$ electrons.

The $\pi$-system in MgP extends over twenty-four centers and includes twenty-six electrons. The inclusion of all the valence $\pi$-orbitals in the active space, which should be the simplest and most straightforward choice, when studying $\pi$ $\rightarrow \pi^{*}$ electronic transitions, is therefore, not possible. However, since we are mainly concerned with the lower energy part of the spectrum, it seems feasible to treat some of the deepest $\pi$-orbitals as inactive and to move some of the weakly occupied orbitals to the virtual space. This way of proceeding is supported by the previous CASPT2 studies on free base porphin. ${ }^{23,36}$

In order to determine the stability of the computed excitation energies with respect to the active space chosen, several active spaces were used. The active space will be represented by means of the notation $O / E$, where the information separated by a slash corresponds to the number of active orbitals and the number of active electrons, respectively. A more detailed description is given in Table II, where it is shown how many orbitals of each symmetry form the active space. In order to provide an idea of the size of the calculations, Table II also includes the number of configuration state functions and determinants obtained for the CASSCF wave function of the ground state with the different active spaces. It should be emphasized here that the CASSCF wave function constitutes the reference function in the second order perturbation treatment where all the electrons, except the core, are correlated within the space spanned by the basis set.

The active space $4 / 4$ contains the two highest occupied molecular orbitals, HOMO and HOMO-1, and the two lowest unoccupied molecular orbitals, LUMO and LUMO +1 , that is, the orbitals involved in the four-orbital model. ${ }^{12} \mathrm{Al}-$ though it has been clearly established that configurations involving orbitals outside the four-orbital model are important for the description of the states ${ }^{36}$ the $4 / 4$ results are included here for the sake of comparison. The eight highest occupied molecular orbitals and the four lowest virtual form the active space 12/16, which has been selected on the basis of the HF orbital energies, as in previous studies of systems with a large number of valence $\pi$-orbitals. ${ }^{37,38}$ Subsequent extensions of the active space have been based on the occupation numbers of the natural orbitals determined with the restricted active space (RAS)SCF method, ${ }^{39,40}$ following the approach used in the first CASPT2 study of free base porphin. ${ }^{36}$

The RASSCF calculations involved all the valence $\pi$-orbitals, 25/26, (since the $3 p_{\pi}$ orbital of the $\mathrm{Mg}$ atom was also considered) partitioning the active space as follows: Eleven orbitals in RAS1, where at most two holes are allowed, four in RAS2, corresponding to the active space $4 / 4$ mentioned above, and ten in RAS3, with the occupation restricted to zero, one, or two. A state-average RASSCF calculation was performed for the optically allowed excited states. Based on these results, at most one occupied orbital and two virtual orbitals, those having the largest deviation from two (occupied) or zero (virtual) in their occupation numbers, were sequentially added to the active space $12 / 16$, resulting in the active spaces 13/18, 14/16, and 15/18 (see Table II). Thus, the largest active space used in the present study, 15/18, contains all the orbitals with occupation numbers in the interval $0.030-1.975$ for any of the studied excited states.

The calculated excitation energies are referred to the ground state computed with the same active space as the excited state. The oscillator strengths were evaluated following the usual procedure: ${ }^{33}$ Transition moments computed at the CASSCF level, by means of the CASSCF state interaction (CASSI) method, ${ }^{41}$ and excitation energies estimated at the CASPT2 level.

The CASSCF/CASPT2 calculations have been performed with the MOLCAS-4 quantum chemistry software ${ }^{42}$ on IBM RS/6000 workstations. The DFT package of the GAUSSIAN 94 program $^{43}$ was used for the geometry optimization.

\section{RESULTS AND DISCUSSION}

We shall start this section considering the HF molecular orbital energies of the molecule since they constitute generally a good tool to get a first insight into the nature of the excited states. The HF energies and the symmetries (in the $D_{4 h}$ symmetry group) of the highest occupied and lowest virtual $\pi$-orbitals are shown in Fig. 1. The lowest oneelectron promotions related to the optically allowed ${ }^{1} E_{u}$ states are also indicated in Fig. 1.

The $\pi$-orbitals of $\mathrm{MgP}$ have a very small contribution coming from the magnesium atomic orbitals and keep similar to those of free base porphin. The orbital energies are of course affected by the $\mathrm{Mg}$ atom which causes, for instance, through the increased symmetry, the $b_{2 g}$ and $b_{3 g}$ orbitals to be degenerate. On the other hand, the net charge on the $\mathrm{Mg}$ atom is computed to be +1.10 at the CASSCF level (independently of the active space used). Back donation from the macrocycle to the vacant $d$ orbitals of the metal explains most of the deviation from the value +2 expected for a $\mathrm{Mg}^{+2}-P^{-2}$ complex. The lower value for the first ionization potential of $\mathrm{MgP}$ in comparison with $\mathrm{FBP}^{44}$ can be rationalized by the higher negative charge in the macrocycle and the increase of the HOMO energy.

As can be seen in Fig. 1, the HOMO and HOMO-1 orbitals are close in energy and quite separated from the remaining occupied orbitals, with a computed gap of $2.7 \mathrm{eV}$. In addition, the two lowest virtual orbitals, which are degenerate, are low in energy. This structure is the basis for the 


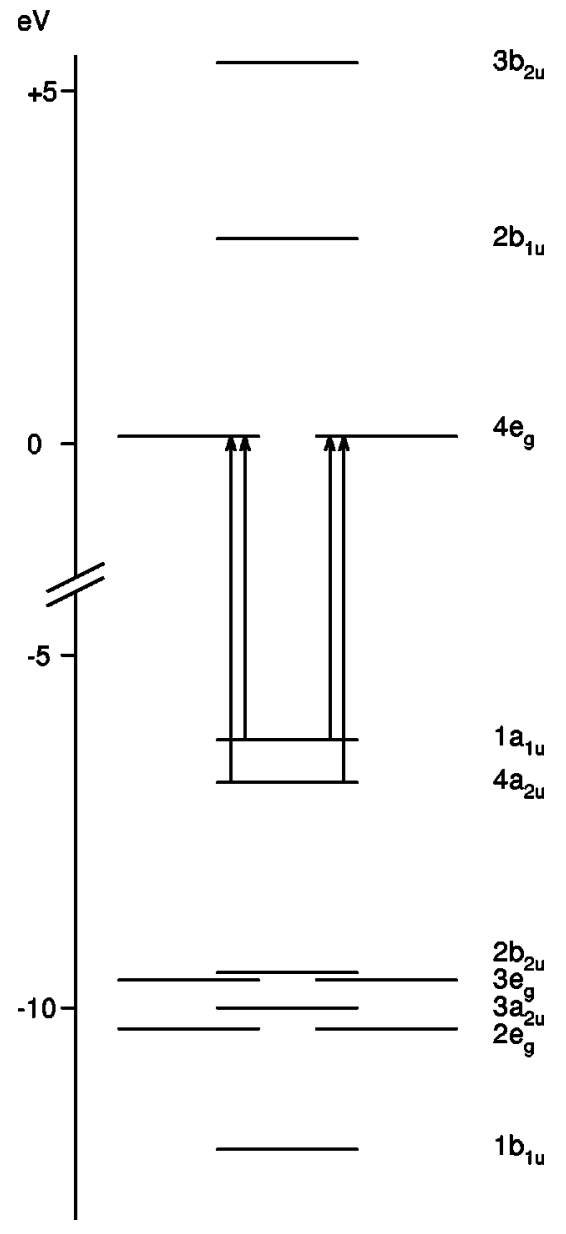

FIG. 1. HF orbital energies of the highest occupied and lowest virtual $\pi$-orbitals of magnesium-porphyrin and a schematic representation of the lowest transitions (the four-orbital model).

four-orbital model. ${ }^{12}$ Within this model, there are two oneelectron promotions with similar energies: The excitations $1 a_{1 u} \rightarrow 4 e_{g}$ and $4 a_{2 u} \rightarrow 4 e_{g}$. Considering the similar energies estimated for both excitations on the basis of the orbital energy differences, the two lowest excited states are expected to be mainly described by a linear combination of them. The "minus" combination has the lower energy and intensity, while the "plus" state is pushed up and carries most of the intensity. This qualitative description is, therefore, able to rationalize the relative intensities of the $Q$ and $B$ bands.

When lower occupied $\pi$-orbitals are taken into account, two higher optically allowed ${ }^{1} E_{u}$ states are predicted. These states are expected to arise from the one-electron promotions $2 b_{2 u} \rightarrow 4 e_{g}$ and $3 a_{2 u} \rightarrow 4 e_{g}$ which could be mixed to some extent. As indicated above, the eight highest occupied $\pi$-orbitals and the four lowest virtual $\pi$-orbitals form the active space $12 / 16$, which contains therefore, the orbitals involved in the description of the four lowest ${ }^{1} E_{u}$ states.

These four lowest ${ }^{1} E_{u}$ states have been computed using the active spaces listed in Table II. The main configurations of the CASSCF wave functions calculated with the largest active space are given in Table III. The weight of the most important configurations involved in the description of the states has been found to be only little affected by the active
TABLE III. Main configurations of the CASSCF wave functions for the ground and ${ }^{1} E_{u}$ states of Mg-Porphyrin. ${ }^{a}$

\begin{tabular}{|c|c|c|c|c|}
\hline State & Main configurations & Weight $(\%)$ & $\mathrm{S}(\%)^{\mathrm{b}}$ & $\mathrm{D}(\%)^{\mathrm{c}}$ \\
\hline $1{ }^{1} A_{1 g}$ & $\cdots\left(4 a_{2 u}\right)^{2}\left(1 a_{1 u}\right)^{2}$ & 80 & $\cdots$ & $14(9)$ \\
\hline \multirow[t]{2}{*}{$1{ }^{1} E_{u}$} & $4 a_{2 u} \rightarrow 4 e_{g}$ & 39 & $4(76)$ & $16(8)$ \\
\hline & $1 a_{1 u} \rightarrow 4 e_{g}$ & 36 & & \\
\hline \multirow[t]{3}{*}{$2{ }^{1} E_{u}$} & $4 a_{2 u} \rightarrow 4 e_{g}$ & 27 & $6(70)$ & $11(8)$ \\
\hline & $1 a_{1 u} \rightarrow 4 e_{g}$ & 35 & & \\
\hline & $3 a_{2 u} \rightarrow 4 e_{g}$ & 6 & & \\
\hline $3{ }^{1} E_{u}$ & $2 b_{2 u} \rightarrow 4 e_{g}$ & 61 & $5(67)$ & $18(14)$ \\
\hline $4{ }^{1} E_{u}$ & $3 a_{2 u} \rightarrow 4 e_{g}$ & 57 & $5(63)$ & $18(17)$ \\
\hline
\end{tabular}

${ }^{a}$ Results from calculations with the active space $15 / 18$.

${ }^{\mathrm{b}}$ Number and weight of singly excited configurations with coefficients larger than 0.05 .

${ }^{\mathrm{c}}$ Number and weight of doubly excited configurations with coefficients larger than 0.05 .

space used in the calculations. As can be seen in Table III, the CASSCF wave function of each excited state is dominated by the singly excited configurations predicted on the basis of the HF orbital energies. However, it should be noticed that these configurations represent only around the $60 \%$ of the CASSCF wave function, except for the $1{ }^{1} E_{u}$ state where they have a total weight of $75 \%$.

Table IV collects the CASSCF and CASPT2 vertical excitation energies and oscillator strengths computed for the four lowest ${ }^{1} E_{u}$ states with the different active spaces. The CASPT2 results corresponding to the active space 15/18 were obtained using a level-shift added to the zeroth-order Hamiltonian in order to remove weakly interacting intruder states which appeared for the two highest states. ${ }^{45} \mathrm{~A}$ value of 0.1 a.u. was used for the level-shift. For the sake of comparison, the experimental values determined from the gas-phase absorption spectra of two different substituted magnesiumporphyrins ${ }^{16,17}$ are also included in Table IV. As far as we know, the absorption spectrum of $\mathrm{MgP}$ itself has not been reported. The influence of the active space on the CASPT2 computed excitation energies will be discussed first. Next, the results will be related to the available experimental data.

As expected on the basis of the study of free base porphin, ${ }^{36}$ the active space $4 / 4$ leads to a too low CASPT2 excitation energy for the $1^{1} E_{u}$ state compared with the results obtained with the larger active spaces and experimental data (see Table IV). In contrast, the CASPT2(4/4) result for the $2{ }^{1} E_{u}$ state deviates only $0.05 \mathrm{eV}$ from the one computed with the largest active space. It is somewhat surprising that the excitation energy of the $2{ }^{1} E_{u}$ state is only slightly affected by the active space used in the calculations. The agreement between these results must be considered fortuitous since the percentage of configurations within the fourorbital model in the CASSCF wave function $(15 / 18)$ of the $2{ }^{1} E_{u}$ state is only $62 \%$, and it is smaller than for the $1{ }^{1} E_{u}$ state, as can be deduced from Table III. As the results collected in Table IV show, the extension of the active space from $12 / 16$ to $15 / 18$ leads to an increase of the CASPT2 excitation energies, which is less than $0.2 \mathrm{eV}$ for the $1^{1} E_{u}$ and $4{ }^{1} E_{u}$ states. The effect is a bit larger for the $3{ }^{1} E_{u}$ state, where a continuous increase of the excitation energy is obtained when the active space is systematically extended. 
TABLE IV. Calculated excitation energies (eV) and oscillator strengths $(f)$ for the optically allowed excited states of Mg-Porphyrin compared to the experimental data.

\begin{tabular}{|c|c|c|c|c|c|c|c|}
\hline \multirow[b]{2}{*}{ State } & \multirow[b]{2}{*}{$(O / E)^{\mathrm{a}}$} & \multirow[b]{2}{*}{ CASSCF } & \multirow[b]{2}{*}{ PT2 } & \multirow[b]{2}{*}{$f$} & \multicolumn{2}{|c|}{ Experiment } & \multirow[b]{2}{*}{ Assignment } \\
\hline & & & & & Mg-Etio ${ }^{\mathrm{b}}$ & $\mathrm{Mg}-\mathrm{TPP}^{\mathrm{c}}$ & \\
\hline \multirow{5}{*}{$1{ }^{1} E_{u}$} & $4 / 4$ & 3.59 & 1.45 & 0.021 & 2.14 & 2.07 & $Q$ \\
\hline & $12 / 16$ & 3.20 & 1.62 & 0.011 & & & \\
\hline & $13 / 18$ & 3.05 & 1.71 & 0.008 & & & \\
\hline & $14 / 16$ & 3.11 & 1.66 & 0.004 & & & \\
\hline & $15 / 18^{\mathrm{d}}$ & 3.06 & 1.78 & $\cdots$ & & & \\
\hline \multirow[t]{5}{*}{$2{ }^{1} E_{u}$} & $4 / 4$ & 5.29 & 2.60 & 1.395 & 3.18 & 3.05 & $B$ \\
\hline & $12 / 16$ & 4.95 & 2.66 & 0.702 & & & \\
\hline & $13 / 18$ & 4.95 & 2.59 & 0.923 & & & \\
\hline & $14 / 16$ & 4.91 & 2.66 & 0.824 & & & \\
\hline & $15 / 18^{\mathrm{d}}$ & 4.98 & 2.65 & $\ldots$ & & & \\
\hline \multirow[t]{4}{*}{$3{ }^{1} E_{u}$} & $12 / 16$ & 5.17 & 2.87 & 0.272 & (3.4) & & \\
\hline & $13 / 18$ & 5.28 & 2.91 & 0.200 & & & \\
\hline & $14 / 16$ & 5.15 & 3.11 & 0.150 & & & \\
\hline & $15 / 18^{\mathrm{d}}$ & 5.35 & 3.18 & $\cdots$ & & & \\
\hline \multirow[t]{4}{*}{$4{ }^{1} E_{u}$} & $12 / 16$ & 5.68 & 3.38 & 0.561 & 3.81 & 3.97 & $N$ \\
\hline & $13 / 18$ & 5.83 & 3.32 & 0.379 & & & \\
\hline & $14 / 16$ & 5.91 & 3.42 & 0.569 & & & \\
\hline & $15 / 18^{\mathrm{d}}$ & 6.09 & 3.55 & $\ldots$ & & & \\
\hline
\end{tabular}

${ }^{a}$ Number of orbitals/number of electrons included in the active space (see Table II)

${ }^{\mathrm{b}} \mathrm{Gas}$-phase absorption spectrum of Mg-Etioporhyrin I (Ref. 16).

${ }^{\mathrm{C}}$ Gas-phase absorption spectrum of Mg-Tetraphenylporphyrin (Ref. 17).

${ }^{\mathrm{d} C A S P T} 2$ calculations using a level-shift of 0.1 a.u.

However, the difference between the CASPT2(14/16) and CASPT2(15/18) results for the $3{ }^{1} E_{u}$ state is less than 0.1 $\mathrm{eV}$. Therefore, the present results are expected to be converged with respect to the active space used within $0.2 \mathrm{eV}$. This does not imply, of course, that the accuracy of the results is $0.2 \mathrm{eV}$, only that with this basis set and using the CASPT2 approach the results will not improve further.

The most intense feature of the gas-phase absorption spectrum of Mg-Etioporphyrin I (Mg-Etio), reported by Edwards $e$ al. ${ }^{16}$ corresponds to the $B$ band. The $Q$ band has only a low intensity and shows two peaks ascribed to the $(0-0)$ and $(0-1)$ vibrational components. ${ }^{16}$ The oscillator strengths for the $Q$ and $B$ bands were estimated to be in the ratio of 1 to 21 from the absorption spectrum of $\mathrm{Mg}$ tetraphenylporphyrin (Mg-TPP) measured in benzene solution. ${ }^{10}$ Based on the computed excitation energies and oscillator strengths, the assignment of the $Q$ band to the $1{ }^{1} E_{u}$ state is clear, as well as the fact that the $2{ }^{1} E_{u}$ state corresponds to the $B$ band. The $1{ }^{1} E_{u}$ state is located at 1.78 $\mathrm{eV}$ [CASPT2(15/18) result], with an oscillator strength predicted to be around 60-200 times smaller (depending on the active space) than for the $2{ }^{1} E_{u}$ state. This is larger than the experimentally obtained relation. The difference is most likely due to vibrational enhancement of the intensity of the lower state. The $0-0$ component of the $Q$ band has been observed at $\approx 2.1 \mathrm{eV}$ in the gas-phase absorption spectrum of substituted magnesium-porphyrins (see Table IV) ${ }^{16,17}$ The $Q$ band of $\mathrm{MgP}$ has been studied in low-temperature rare gas and nitrogen matrices, where the $0-0$ band is detected at 2.2 $\mathrm{eV}^{46}$

The Soret region ( $B$ band) of the absorption spectrum of
Mg-Etio appears in the energy range 3.1-3.5 eV, with the maximum at $3.18 \mathrm{eV} .{ }^{16}$ The $2{ }^{1} E_{u}$ state is the most intense of the four states studied here. The transition is predicted at 2.7 $\mathrm{eV}$ and should clearly be assigned to the intense $B$ band. According to the best calculation level used in this study, the weaker $3{ }^{1} E_{u}$ state is located $3.18 \mathrm{eV}$ above the ground state. We assign this state to the shoulder at the high energy side of the $B$ band, which appears at $\sim 3.4 \mathrm{eV}$. The $4{ }^{1} E_{u}$ state, computed at $3.55 \mathrm{eV}$ using the largest active space, is thus assigned to the $N$ band, which has the maximum at $3.81 \mathrm{eV}$ in the absorption spectrum of Mg-Etio. ${ }^{16}$

Overall, the computed excitation energies are underestimated $(0.3-0.5 \mathrm{eV})$ with respect to the experimental data coming from substituted magnesium-porphyrins. The same trend, but with smaller deviation, was found in our previous study free base porphin. ${ }^{23}$ However, the proposed assignments for the excited states of $\mathrm{MgP}$ are consistent with the observed spectra in substituted systems. Moreover, they are strongly supported by the recent interpretation of the electronic spectrum of free base porphin, ${ }^{23}$ as we shall discuss below.

The symmetry adapted cluster-configuration interaction (SAC-CI) method has recently been applied to the study of the excited states of MgP by Hasegawa et al. ${ }^{27} \mathrm{~A}$ small basis set, without polarization functions, was used. The SAC-CI results are shown in Table $\mathrm{V}$ together with the CASPT2 excitation energies and oscillator strengths computed using the active space $13 / 18$ and a basis set which lacks polarization functions in order to have a more comparable set of results. The first thing to notice is the increase obtained in the CASPT2 values $(0.2-0.5 \mathrm{eV})$ when the polarization 
TABLE V. CASPT2 excitation energies (eV) and oscillator strengths $(f)$ of the singlet $E_{u}$ states of Mg-Porphyrin compared to other $a b$ initio results. ${ }^{a}$

\begin{tabular}{ccccccc}
\hline \hline & \multicolumn{2}{c}{ BS1 $^{\mathrm{b}}$} & & \multicolumn{2}{c}{$\mathrm{BS2}^{\mathrm{c}}$} & \\
\cline { 2 - 3 } State & CASPT2 & $f$ & & CASPT2 & $f$ & \multicolumn{1}{c}{ SAC-CI $^{\mathrm{d}}$} \\
\hline${ }^{1}{ }^{1} E_{u}$ & 1.71 & 0.008 & & 1.95 & $8 \times 10^{-5}$ & $2.01\left(1.5 \times 10^{-3}\right)$ \\
$2{ }^{1} E_{u}$ & 2.59 & 0.923 & & 2.91 & 1.128 & $3.63(1.99)$ \\
$3{ }^{1} E_{u}$ & 2.91 & 0.200 & & 3.37 & 0.107 & $4.15(0.069)$ \\
${ }^{1}{ }^{1} E_{u}$ & 3.32 & 0.379 & & 3.84 & 0.476 & $4.89(0.590)$ \\
\hline \hline
\end{tabular}

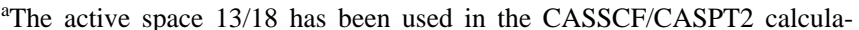
tions.

${ }^{\mathrm{b}} \mathrm{Mg}[4 s 3 p 1 d] / \mathrm{C}, \mathrm{N}[3 s 2 p 1 d] / \mathrm{H}[2 s]$ basis set.

${ }^{\mathrm{c}} \mathrm{Mg}[4 s 3 p 1 d] / \mathrm{C}, \mathrm{N}[3 s 2 p] / \mathrm{H}[2 s]$ basis set.

${ }^{d}$ Reference 27. The computed oscillator strengths are given within parentheses.

functions are excluded of the basis set, which is in line with the influence found in the study of FBP. ${ }^{23}$ Therefore, the lack of polarization functions in the basis set fortuitously improves agreement with experiment. On the other hand, the SAC-CI method yields larger excitation energies than the CASPT2 method for all the states investigated. The difference between both sets of results increases when the energy of the state increases. This is also the trend found when comparing the CASPT2 and SAC-CI results for FBP. ${ }^{23}$

The larger SAC-CI excitation energies compared to the CASPT2 values explains the different assignments made by Hasegawa et al. $^{27}$ Thus, these authors have assigned the $3{ }^{1} E_{u}$ state to the $N$ band and the $4{ }^{1} E_{u}$ state to the $M$ band with a maximum observed at $5.8 \mathrm{eV}$ in $\mathrm{Mg}$-Etio ${ }^{16}$ and at 6.2 $\mathrm{eV}$ in Mg-TPP. ${ }^{17}$ With this assignment, the SAC-CI results agree with the experimental data for the three lowest ${ }^{1} E_{u}$ states (with a tendency to overestimate the energies), while the SAC-CI excitation energy for the $4{ }^{1} E_{u}$ state is now lower than experiment (computed value $4.9 \mathrm{eV}$, experiment in the region around $6 \mathrm{eV}$ ). Considering the SAC-CI results for free base porphin, ${ }^{21}$ the computed excitation energies, and therefore, the conclusions reached in the SAC-CI study, ${ }^{27}$ are not expected to be considerably modified by further extensions of the one-electron basis set. The assignments proposed in the present study, with the second and third ${ }^{1} E_{u}$ states related to the $B$ band and the $4{ }^{1} E_{u}$ state ascribed to the $N$ band, lead to a consistent set of results, not only with the experimental information but also between them, in the sense that deviation from experiments is similar for all states. The same interpretation of the electronic spectrum of $\mathrm{MgP}$ as the one suggested here was provided in the first $a b$ initio study performed by Petke et al. ${ }^{26}$ in 1978. At that time, the computed excitation energies had to be fitted, using the theoretical and experimental values coming from clear assignments, in order to get a better estimate of the transition energies and make the assignments possible. ${ }^{26}$

We now address the question of how the interpretation given in the present work for the electronic spectrum of $\mathrm{MgP}$ is supported by the results obtained for the excited states of free base porphin in our previous study. ${ }^{23}$ To discuss this point, we recall some of the results. The excitation energies and oscillator strengths previously computed for FBP are collected in Table VI together with the present results for $\mathrm{MgP}$. In FBP, due to the lower symmetry, the ${ }^{1} E_{u}$ states are split into ${ }^{1} B_{3 u}$ and ${ }^{1} B_{2 u}$. According to the CASPT2 results, the splitting is, however, only $\approx 0.1 \mathrm{eV}$, except for the first pair of states where the computed energy difference is 0.44 $\mathrm{eV}$, in agreement with the gas-phase experimental data. ${ }^{17}$ The $B$ band appears in FBP broader than in MgP (and than in metal-porphyrins) with a pronounced shoulder in the high energy tail, which corresponds to the so called $N$ band in this system. ${ }^{17}$ As indicated in Table VI, the $B$ band is assigned to the $2{ }^{1} B_{3 u}$ and $2{ }^{1} B_{2 u}$ states and the $N$ band corresponds to the third pair of states. These four states are predicted to be responsible for the intensity of the $B-N$ band in porphin. The assignment of the $2{ }^{1} E_{u}$ and $3{ }^{1} E_{u}$ states of $\mathrm{MgP}$ to the $B$ band, with the $3{ }^{1} E_{u}$ state in the high energy tail, leads therefore, to the same description of this part of the spectrum as in porphin. The difference seems to be then a question of the

TABLE VI. Calculated excitation energies (eV) and oscillator strengths $(f)$ for the lowest singlet $\pi \rightarrow \pi^{*}$ optically allowed excited states of free base porphin and Mg-Porphyrin. ${ }^{\mathrm{a}}$

\begin{tabular}{|c|c|c|c|c|c|c|c|}
\hline \multirow[b]{2}{*}{ State } & \multicolumn{4}{|c|}{$\mathrm{FBP}^{\mathrm{b}}$} & \multicolumn{3}{|c|}{$\mathrm{MgP}$} \\
\hline & $\mathrm{PT} 2^{\mathrm{c}}$ & $f$ & Experiment $^{\mathrm{d}}$ & & PT2 & $f$ & \\
\hline $1^{1} B_{3 u}$ & 1.63 & 0.004 & $1.98^{\mathrm{e}}$ & $Q_{x}$ & & م 00 & \\
\hline $\begin{array}{l}1{ }^{1} B_{2 u} \\
2{ }^{1} B_{2 u}\end{array}$ & $\begin{array}{l}2.11 \\
3.08\end{array}$ & $\begin{array}{l}0.002 \\
0.911\end{array}$ & $2.42^{\mathrm{e}}$ & $Q_{y}$ & 1.66 & 0.004 & $Q$ \\
\hline $\begin{array}{l}2{ }^{1} B_{3 u} \\
3{ }^{1} B_{2 u}\end{array}$ & $\begin{array}{l}3.12 \\
3.42\end{array}$ & $\begin{array}{l}0.704 \\
0.458\end{array}$ & 3.33 & $B$ & 2.66 & 0.824 & $B$ \\
\hline $\begin{array}{l}3{ }^{1} B_{3 u} \\
4{ }^{1} B_{2}\end{array}$ & $\begin{array}{l}3.53 \\
3.96\end{array}$ & $\begin{array}{l}0.833 \\
0.341\end{array}$ & 3.65 & $N$ & 3.11 & 0.150 & \\
\hline $4{ }^{1} B_{3 u}$ & 4.04 & 0.202 & 4.25 & $L$ & 3.42 & 0.569 & $N$ \\
\hline
\end{tabular}

${ }^{a}$ Results from calculations with the active space 14/16.

${ }^{\mathrm{b}}$ Reference 23.

${ }^{\mathrm{c} C a l c u l a t i o n s}$ using a level-shift of 0.4 a.u.

${ }^{\mathrm{d}}$ Gas-phase absorption spectrum (Ref. 17).

${ }^{\mathrm{e}} 0-0$ band. 
TABLE VII. Computed excitation energies (eV) of the optically forbidden $\pi-\pi^{*}$ excited states of Mg-Porphyrin.

\begin{tabular}{cccc}
\hline \hline State & O/E & CASSCF & CASPT2 \\
\hline $1{ }^{1} A_{2 g}$ & $13 / 18$ & 5.14 & 2.75 \\
& $14 / 18$ & 5.13 & 2.72 \\
$1{ }^{1} B_{2 g}$ & $13 / 18$ & 4.77 & $\ldots$ \\
& $14 / 18$ & 4.65 & 2.78 \\
$2{ }^{1} B_{2 g}$ & $13 / 18$ & 5.15 & 2.67 \\
& $14 / 18$ & 4.94 & 2.78 \\
${ }^{1}{ }^{1} B_{1 g}$ & $13 / 18$ & 5.34 & 3.08 \\
& $14 / 18$ & 5.25 & 3.05 \\
$2{ }^{1} B_{1 g}$ & $13 / 18$ & 4.48 & $\cdots$ \\
& $14 / 18$ & 4.43 & $3.29^{\mathrm{a}}$ \\
$2{ }^{1} A_{2 g}$ & $13 / 18$ & 5.53 & 3.22 \\
& $14 / 18$ & 5.29 & 3.31 \\
$2{ }^{1} A_{1 g}$ & $13 / 18$ & 4.91 & 3.55 \\
& $14 / 18$ & 4.93 & 3.38 \\
$3{ }^{1} B_{2 g}$ & $13 / 18$ & 5.76 & 3.34 \\
& $14 / 18$ & 5.62 & 3.50 \\
\hline \hline
\end{tabular}

${ }^{\mathrm{a} A}$ level-shift of 0.2 a.u. was used.

names used for the bands, since the band called $N$ in magnesium-porphyrins, assigned to the $4{ }^{1} E_{u}$ state, appears separated from the $B$ band. That $N$ band could better be related to the $L$ band in porphin, as the similar description of the corresponding states suggests (see Table III and Table II in Ref. 23). The general similarity between the electronic spectra of porphin and metal-porphyrins gives support to these suggestions.

On the other hand, the narrower Soret band in magnesium-porphyrins compared to porphin can be attributed not only to the increase of the symmetry but also to the smaller values estimated for the intensity of the transition to the $3{ }^{1} E_{u}$ state, as indicated by the oscillator strengths reported in Table VI. The different intensities for the third state in the two systems can be rationalized by the differences found in the CASSCF wave functions of the states. Thus, the $3{ }^{1} B_{3 u}$ state of FBP is mainly described by the one-electron promotions $4 b_{1 u} \rightarrow 4 b_{2 g}$ and $5 b_{1 u} \rightarrow 4 b_{2 g}$, with weights of $29 \%$ and $22 \%$, respectively, while the ${ }^{1} B_{3 u}$ component of the $3{ }^{1} E_{u}$ state of $\mathrm{MgP}$ is dominated by the singly excited configuration $5 b_{1 u} \rightarrow 4 b_{2 g}$ (61\%). It should be clarified that the $5 b_{1 u}$ orbital of $\mathrm{MgP}$ corresponds to the $4 b_{1 u}$ orbital of FBP. The $3{ }^{1} B_{3 u}$ state of FBP shows, therefore, some mixing with the intense $2{ }^{1} B_{3 u}$ state through the singly excited configuration $5 b_{1 u} \rightarrow 4 b_{2 g}$.

Finally, we present in Table VII the computed excitation energies for the optically forbidden $\pi \rightarrow \pi^{*}$ states of magnesium-porphyrin. The states up to $3.5 \mathrm{eV}$ have been considered. A first set of calculations were performed using the active space $13 / 18$, which was subsequently extended with an extra virtual orbital (active space 14/18 in Table VII), following the CASPT2 requirements found for two of the states studied. As expected, the CASPT2 excitation energies are slightly affected by the extension of the active space, excluding of course the states where that extension was needed in order for the state to be included in the CASSCF configuration space. The influence of the active space is, however, more important for the two highest states, but still the changes in the excitation energies are less than
TABLE VIII. Calculated excitation energies (eV) for the two lowest triplet $E_{u}$ states of Mg-Porphyrin.

\begin{tabular}{ccccc}
\hline \hline State & $(O / E)^{\mathrm{a}}$ & CASSCF & CASPT2 & Experiment \\
\hline $1^{3} E_{u}$ & $4 / 4$ & 3.24 & 1.12 & \\
& $12 / 16$ & 2.73 & 1.46 & \\
& $13 / 18$ & 2.58 & 1.57 & $1.7^{\mathrm{b}} 1.6^{\mathrm{c}}$ \\
& $14 / 16$ & 2.54 & 1.55 & \\
$2{ }^{3} E_{u}$ & $4 / 4$ & 2.70 & 1.54 & \\
& $12 / 16$ & 2.43 & 1.82 & \\
& $13 / 18$ & 2.38 & 1.92 & \\
& $14 / 16$ & 2.41 & 1.79 & \\
\hline \hline
\end{tabular}

${ }^{a}$ Number of orbitals/number of electrons included in the active space (see Table II).

${ }^{\mathrm{b}} \mathrm{Phosphorescence} \mathrm{spectrum} \mathrm{of} \mathrm{magnesium} \mathrm{etioporphyrin} \mathrm{II} \mathrm{in} \mathrm{EPA} \mathrm{glass}$ (Ref. 11).

${ }^{c}$ Phosphorescence spectrum in (EPA) glass; sharp peak with maximum at $790 \mathrm{~nm}$ (Ref. 10).

$0.2 \mathrm{eV}$. As can be seen in Table VII, the lowest optically forbidden state appears at $2.7 \mathrm{eV}$, that is in the Soret region of the spectrum. Theoretical studies of free base porphin ${ }^{21,22}$ have also predicted that the forbidden states start to appear in the Soret region. The present results find eight optically forbidden $\pi \rightarrow \pi^{*}$ states in the energy range $2.7-3.5 \mathrm{eV}$, as shown in Table VII. As regards the CASSCF description of the states, they are mainly characterized by excitations to the double degenerate LUMO orbital, except for the $1{ }^{1} B_{2 g}$ and $2{ }^{1} B_{1 g}$ states where the LUMO+1 orbital has an important contribution.

The two lowest triplet states of $E_{u}$ symmetry have also been studied using different active spaces. The results are collected in Table VIII together with the experimental data coming from the phosphorescence spectra. ${ }^{10,11}$ As can be seen in the table, the active space $4 / 4$ provides too low CASPT2 excitation energies while the values obtained with the larger active spaces seem to be converged to $\approx 0.1 \mathrm{eV}$. The lowest triplet state, $1{ }^{3} E_{u}$, is located at $1.55 \mathrm{eV}$ above the ground state at the CASPT2(14/16) level, in agreement with the energy $1.6-1.7 \mathrm{eV}$ determined from the phosphorescence spectra in EPA glass. ${ }^{10,11}$ The most extensive calculation places the $2{ }^{3} E_{u}$ state at $1.79 \mathrm{eV}$, that is, quite close in energy to the $1^{3} E_{u}$ state. The CASSCF description of the two lowest triplet $E_{u}$ states is dominated by one singly excited configuration: The $4 a_{2 u} \rightarrow 4 e_{g}$ with weight $81 \%$ in the $1^{3} E_{u}$ state and the $1 a_{1 u} \rightarrow 4 e_{g}(82 \%)$ for the $2{ }^{3} E_{u}$ state.

\section{SUMMARY}

In this paper, we have reported results for the vertical excitation energies on the low-lying singlet and triplet excited states of magnesium-porphyrin. The investigation has been performed using the CASSCF/CASPT2 methodology and a split-valence polarization basis set and constitutes the highest level of theory used for the study of the excited states of a metal porphyrin so far.

The present results lead to a different assignment of the electronic spectrum compared to the other recent $a b$ initio study. They are in agreement with experiment and also consistent with earlier results obtained for free base porphin us- 
ing the same computational procedure. The four pairs of states found in FBP has in MgP merged into four degenerate states with an energy shifted downwards a few tenths of an $\mathrm{eV}$ with respect to the average excitation energy for the corresponding pair in FBP.

\section{ACKNOWLEDGMENTS}

The research reported in this communication has been supported by the European Commission through the TMR program (Grant No. ERBFMRXCT960079), by the Swedish Natural Science Research Council (NFR), and by project PB97-1377 of the DGES of Spain.

${ }^{1}$ R. W. Wagner, J. S. Lindsey, J. Seth, V. Palaniappan, and D. F. Bocian, J. Am. Chem. Soc. 118, 3996 (1996).

${ }^{2}$ J. R. Reimers, T. X. Lü, M. J. Crossley, and N. S. Hush, Chem. Phys. Lett. 256, 353 (1996).

${ }^{3}$ D. Beljonne, G. E. O'Keefe, P. J. Hamer, R. H. Friend, H. L. Anderson, and J. L. Brédas, J. Chem. Phys. 106, 9439 (1997).

${ }^{4}$ F. Z. Henari, W. J. Blau, L. R. Milgrom, G. Yahioglu, D. Phillips, and J. A. Lacey, Chem. Phys. Lett. 267, 229 (1997).

${ }^{5}$ Z. Wang, P. N. Day, and R. Pachter, J. Chem. Phys. 108, 2504 (1998).

${ }^{6}$ L. Stryer, in Biochemistry (Freeman, New York, 1988).

${ }^{7}$ R. E. Christoffersen, Int. J. Quantum Chem. 16, 573 (1979).

${ }^{8}$ J. D. Petke and G. M. Maggiora, J. Chem. Phys. 84, 1640 (1986).

${ }^{9}$ M. R. Wasielewski, Chem. Rev. 92, 435 (1992).

${ }^{10}$ G. D. Dorough, J. R. Miller, and F. M. Huennekens, J. Am. Chem. Soc. 73, 4315 (1951).

${ }^{11}$ J. B. Allison and R. S. Becker, J. Chem. Phys. 32, 1410 (1960).

${ }^{12}$ M. Gouterman, J. Chem. Phys. 30, 1139 (1959).

${ }^{13}$ W. T. Simpson, J. Chem. Phys. 17, 1218 (1949).

${ }^{14}$ C. Weiss, H. Kobayashi, and M. Gouterman, J. Mol. Spectrosc. 16, 415 (1965).

${ }^{15}$ W. S. Caughey, R. M. Deal, C. Weiss, and M. Gouterman, J. Mol. Spectrosc. 16, 451 (1965).

${ }^{16}$ L. Edwards, D. H. Dolphin, and M. Gouterman, J. Mol. Spectrosc. 35, 90 (1970).

${ }^{17}$ L. Edwards, D. H. Dolphin, M. Gouterman, and A. D. Adler, J. Mol. Spectrosc. 38, 16 (1971).

${ }^{18}$ J. Rodriguez, C. Kirmaier, and D. Holten, J. Am. Chem. Soc. 111, 6500 (1989).

${ }^{19}$ I. Baraldi, A. Carnevali, G. Ponterini, and D. Vanossi, J. Mol. Struct.: THEOCHEM 333, 121 (1995).

${ }^{20}$ A. J. McHugh, M. Gouterman, and C. Weiss, Theor. Chim. Acta 24, 346 (1972).

${ }^{21}$ Y. Tokita, J. Hasegawa, and H. Nakatsuji, J. Phys. Chem. 102, 1843 (1998).

${ }^{22}$ S. R. Gwaltney and R. J. Bartlett, J. Chem. Phys. 108, 6790 (1998).

${ }^{23}$ L. Serrano-Andrés, M. Merchán, M. Rubio, and B. O. Roos, Chem. Phys. Lett. 295, 195 (1998).

${ }^{24}$ C. Weiss, J. Mol. Spectrosc. 44, 37 (1972).
${ }^{25}$ Y. Nonomura, S. Igarashi, N. Yoshioka, and H. Inoue, Chem. Phys. 220, 155 (1997).

${ }^{26}$ J. D. Petke, G. M. Maggiora, L. L. Shipman, and R. E. Christoffersen, J. Mol. Spectrosc. 71, 64 (1978).

${ }^{27}$ J. Hasegawa, M. Hada, M. Nonoguchi, and H. Nakatsuji, Chem. Phys. Lett. 250, 159 (1996).

${ }^{28}$ K. Pierloot, B. Dumez, P.-O. Widmark, and B. O. Roos, Theor. Chim. Acta 90, 87 (1995).

${ }^{29}$ N. Matsuzawa, M. Ata, and D. A. Dixon, J. Phys. Chem. 99, 7698 (1995).

${ }^{30}$ D. Lamoen and M. Parrinello, Chem. Phys. Lett. 248, 309 (1996).

${ }^{31}$ K. Andersson, P.-A. Malmqvist, B. O. Roos, A. J. Sadlej, and K. Wolinski, J. Phys. Chem. 94, 5483 (1990).

${ }^{32}$ K. Andersson, P.-A. Malmqvist, and B. O. Roos, J. Chem. Phys. 96, 1218 (1992).

${ }^{33}$ B. O. Roos, M. P. Fülscher, Per-Åke Malmqvist, M. Merchán, and L. Serrano-Andrés, in Quantum Mechanical Electronic Structure Calculations with Chemical Accuracy, edited by S. R. Langhoff (Kluwer Academic, Dordrecht, The Netherlands, 1995), p. 357.

${ }^{34}$ B. O. Roos, K. Andersson, M. P. Fülscher, P.A. Malmqvist, L. SerranoAndrés, K. Pierloot, and M. Merchán, in Advances in Chemical Physics: New Methods in Computational Quantum Mechanics, Vol. XCIII:219, edited by I. Prigogine and S. A. Rice (Wiley, New York, 1996).

${ }^{35}$ B. O. Roos, in Advances in Chemical Physics; Ab Initio Methods in Quantum Chemistry - II, edited by K. P. Lawley (Wiley, Chichester, England, 1987), Chap. 69, p. 399.

${ }^{36}$ M. Merchán, E. Ortí, and B. O. Roos, Chem. Phys. Lett. 226, 27 (1994).

${ }^{37}$ M. Rubio, M. Merchán, E. Ortí, and B. O. Roos, Chem. Phys. Lett. 248, 321 (1996).

${ }^{38}$ V. Molina, M. Merchán, and B. O. Roos, J. Phys. Chem. A 101, 3478 (1997).

${ }^{39}$ J. Olsen, B. O. Roos, P. Jørgensen, and H. J. Aa. Jensen, J. Chem. Phys. 89, 2185 (1988).

${ }^{40}$ P.-Å. Malmqvist, A. Rendell, and B. O. Roos, J. Phys. Chem. 94, 5477 (1990).

${ }^{41}$ P.-Å. Malmqvist and B. O. Roos, Chem. Phys. Lett. 155, 189 (1989).

${ }^{42}$ MOLCAS, Version 4.0, K. Andersson, M. R. A. Blomberg, M. P. Fülscher, G. Karlstöm, R. Lindh, P.-Å. Malmqvist, P. Neogrády, J. Olsen, B. O. Roos, A. J. Sadlej, M. Schütz, L. Seijo, L. Serrano-Andrés, P. E. M. Siegbahn, and P.-O. Widmark, Dept. of Theor. Chem., Chem. Center, Univ. of Lund, P.O.B. 124, S-221 00 Lund, Sweden, Lund, 1997.

${ }^{43}$ Gaussian 94, M. J. Frisch, G. W. Trucks, H. B. Schlegel, P. M. W. Gill, B. G. Johnson, M. A. Robb, J. R. Cheeseman, T. Keith, G. A. Petersson, J. A. Montgomery, K. Raghavachari, M. A. Al-Laham, V. G. Zakrzewski, J. V. Ortiz, J. B. Foresman, J. Cioslowski, B. B. Stefanov, A. Nanayakkara, M. Challacombe, C. Y. Peng, P. Y. Ayala, W. Chen, M. W. Wong, J. L. Andres, E. S. Replogle, R. Gomperts, R. L. Martin, D. J. Fox, J. S. Binkley, D. J. Defrees, J. Baker, J. P. Stewart, M. Head-Gordon, C. Gonzalez, and J. A. Pople, Gaussian, Inc., Pittsburgh, PA, 1995.

${ }^{44}$ P. Dupuis, R. Roberge, and C. Sandorfy, Chem. Phys. Lett. 75, 434 (1980).

${ }^{45}$ B. O. Roos and K. Andersson, Chem. Phys. Lett. 245, 215 (1995).

${ }^{46}$ A. Starukhin, A. Shulga, and J. Waluk, Chem. Phys. Lett. 272, 405 (1997). 